\title{
CALIBRACIÓN Y APLICACIÓN DE UN MODELO DE EROSIÓN Y DINÁMICA DEL CARBONO (SPEROS-C) A DOCE PEQUEÑAS CUENCAS DEL SURESTE ESPAÑOL
}

\author{
E. NADEU1,2*, K. VAN OOST2, \\ J. DE VENTE' ${ }^{1}$, C. BOIX-FAYOS ${ }^{1}$ \\ ${ }^{1}$ Departamento de Conservación de Suelos y Aguas y Manejo de Residuos Orgánicos, CEBAS-CSIC, \\ Campus Universitario de Espinardo, Apartado de Correos 164, 30100 Murcia, España. \\ ${ }^{2}$ Georges Lemaitre Centre for Earth and Climate Research, Earth and Life Institute, \\ Université catholique de Louvain, B-1348 Louvain-la-Neuve, Bélgica.
}

RESUMEN. Varios estudios publicados durante las últimas décadas indican que la erosión del suelo puede estar jugando un papel importante en el ciclo global del carbono $(C)$. Una de las mayores incertidumbres en la actualidad está en el efecto que los flujos laterales de sedimento y $C$ ejercen sobre la dinámica del carbono orgánico del suelo y su redistribución en el paisaje. Para su estudio desde una perspectiva de modelización, es necesaria la aplicación de un modelo que simule procesos de redistribución de sedimento y $C$ y, a la vez, represente los cambios que ocurren a nivel de perfil de suelo cuando el C se pierde del perfil por la erosión del suelo o es ganado por deposición. Sin embargo, son pocos los modelos que actualmente pueden simular estos procesos y se han aplicado mayoritariamente en condiciones de paisaje homogéneas. Aquí adaptamos, calibramos y validamos un modelo (SPEROS-C) utilizado con éxito en otras zonas para estimar los flujos de C inducidos por los procesos erosivos en 12 cuencas (6-500 ha) heterogéneas en vegetación, litología y topografía. Los resultados muestran tasas de erosión de C similares a las obtenidas en otros estudios. En el contexto del ciclo del C, el balance entre la pérdida, el entierro y el reemplazo de $C$ en las cuencas resultó en la mayoría de los casos en un secuestro de $C$ durante el periodo modelizado (28 años). Estos resultados indican que a pesar de sus múltiples efectos negativos sobre los ecosistemas, la erosión del suelo puede inducir el secuestro de C en determinados casos y horizontes temporales.

\section{Calibration and application of an erosion and $C$ redistribution model} (SPEROS-C) to twelve small catchments in southeastern Spain

ABSTRACT. Results from various studies published during the last decades suggest that soil erosion could be playing an important role in the global carbon (C) cycle. One of the major uncertainties is related to the effect that lateral fluxes of sediment and $C$ may have on soil $C$ dynamics and on its distribution within the 
landscape. To approach its study from a modeling perspective, a model simulating redistribution processes of sediment and $C$, as well as the changes taking place within the soil profile when $C$ is removed or deposited, is needed. However, not many models can currently perform these simulations, and the existing ones have been applied mainly to very homogeneous landscapes. Here we adapted, calibrated and validated a soil erosion and $C$ redistribution model (SPEROS-C), successfully used in other regions, in order to estimate erosioninduced $C$ fluxes in 12 small catchments (6-500 ha). These catchments were heterogeneous from a vegetation, lithology and topographical perspective. The estimated erosion and $C$ redistribution rates were similar to those obtained in other studies. In the context of the $C$ cycle, the balance between $C$ loss, burial and replacement in eroded slopes in the catchment resulted, for most catchments, in a net $C$ sequestration during the studied period (28 years). These results indicate that despite its multiple negative effects on ecosystems, soil erosion can induce $C$ sequestration under specific conditions and temporal scales.

Palabras clave: erosión del suelo, modelización, redistribución de carbono, dique de retención, cuenca.

Key words: soil erosion, modeling, carbon redistribution, check-dam, catchment.

Enviado el 5 de noviembre de 2012

Aceptado el 12 de enero de 2013

* Correspondencia: Departamento de Conservación de Suelos y Aguas y Manejo de Residuos Orgánicos, CEBAS-CSIC, Campus Universitario de Espinardo, Apartado de Correos 164, 30100 Murcia. E-mail: elisabet.nadeu@uclouvain.be

\section{Introducción}

Los procesos de erosión hídrica inducen la movilización de carbono orgánico del suelo (SOC) a través de flujos laterales de carbono (C) (redistribución en superficie) y flujos verticales de $\mathrm{C}$ (intercambio de $\mathrm{C}$ entre la atmósfera y el suelo a través de procesos de mineralización o secuestro de C). En la actualidad se estima que la combinación de flujos laterales y verticales de $\mathrm{C}$ inducidos por la erosión, puede tener un impacto significativo sobre el balance global de $\mathrm{C}$ como resultado de la interacción de varios procesos (Berhe et al., 2007; Van Oost et al., 2007). Los procesos erosivos redistribuyen sedimento y, junto a ellos, nutrientes por el paisaje, mientras que una pequeña parte del material movilizado llega a cursos fluviales (Chaplot et al., 2005; Van Oost et al., 2007). El C depositado en sistemas terrestres puede ser almacenado y protegido de procesos de descomposición o bien mineralizado a la atmósfera, como se ha mostrado en experimentos de campo y laboratorio (Polyakov y Lal, 2004; Van Hemelryck et al., 2011). En el perfil de suelo erosionado, la movilización de sedimento y $\mathrm{C}$ provoca la exposición de C más estable que se encontraba en horizontes más profundos, disminuyendo así las tasas de respiración del suelo y posibilitando también la entrada de materia orgánica 
fresca, en el proceso conocido como reemplazo de C (Harden et al., 1999). Los procesos erosivos pueden, pues, alterar el ciclo biogeoquímico del $\mathrm{C}$ incrementando o disminuyendo las reservas de $\mathrm{C}$ en el suelo, y acelerando o ralentizando la descomposición del C movilizado (Gregorich et al., 1998). Una mejor comprensión del comportamiento de los flujos de $\mathrm{C}$ laterales es crucial en la actualidad para determinar el papel de los mismos en la redistribución del $\mathrm{C}$ en el paisaje y en el balance de $\mathrm{C}$ en cuencas de drenaje y a nivel global.

La modelización es una buena herramienta para simular los flujos de carbono orgánico inducidos por erosión y ayudar a la evaluar la importancia de los mismos en el marco de la escala de cuenca, donde determinadas clases y patrones de cobertura vegetal y uso del suelo interactúan en la dinámica erosiva y deposicional. Desafortunadamente, no existen muchos modelos actualmente que puedan realizar estas simulaciones combinando flujos horizontales y verticales de movilización de carbono. Los primeros modelos utilizados en este sentido fueron los que actúan a escala de perfil de suelo, simulando la dinámica de $\mathrm{C}$ en diferentes horizontes de suelo y entre los mismos, reproduciendo los efectos locales de erosión y deposición en el mismo perfil, pero sin evaluar la redistribución espacial de C. Éste es el caso del modelo EDEM (Erosion-DepositionCarbon-Model) (Liu et al., 2003), basado en el modelo de dinámica de carbono CENTURY (Parton et al., 1987), o el modelo de hoja de cálculo SOrCERO (Billings et al., 2010). Con el objeto de superar las limitaciones de estos modelos aplicados a perfiles individuales, varias combinaciones de los mismos se han aplicado a áreas más grandes en los últimos años. Desde esta aproximación encontramos la combinación de los modelos SWAT (Arnold et al., 1998), WEPP (Flanagan y Nearing, 1995), y CENTURY aplicados a una cuenca de 12300 hectáreas tratando de evaluar el potencial de diferentes combinaciones de uso de suelo para los programas de créditos de carbono (Yadav et al., 2009), o la aplicación de GeoWEPP (Renschler, 2003), que combinado con CENTURY (Yadav y Malanson, 2009) estudia la dinámica de erosión y deposición bajo diferentes usos del suelo en una cuenca de 28 hectáreas. En pastizales, el modelo CREEP (Changing Relief and Evolving Ecosystems Program) (Rosenbloom et al., 2001) simula los procesos geomorfológicos de difusión que se centran en el movimiento diferencial de las partículas del suelo (y el C adherido a las mismas) y se ha utilizado para comprender y cuantificar los efectos de la erosión del suelo en la acumulación de $\mathrm{C}$ en el paisaje. Aunque la mayoría de estos modelos se ha aplicado en áreas con presencia de zonas agrícolas, el único, hasta el momento, que tiene en cuenta el efecto de la erosión del suelo en combinación con el efecto del laboreo en la redistribución de C es el modelo SPEROS-C (Van Oost et al., 2005). Así, SPEROS-C combina la redistribución de suelo por erosión hídrica y por laboreo (Van Oost et al., 2003) con el modelo ICBM de balance de carbono (Introductory Carbon Balance Model) (Andrén y Kätterer, 1997), que simula flujos verticales de $\mathrm{C}$ dentro del perfil del suelo. SPEROS-C ha sido aplicado con éxito a pequeñas cuencas agrícolas bastante homogéneas en términos de tipos y usos de suelo, litología y pendientes (Dugloss et al., 2012; Van Oost et al., 2005), permitiendo cuantificar el $\mathrm{C}$ exportado y redistribuido en las mismas. 
En este contexto pretendemos con este trabajo avanzar en el conocimiento de la redistribución del carbono por erosión en cuencas de drenaje en ambiente mediterráneo de montaña media a través de dos objetivos concretos. El primero: (i) adaptar, calibrar y validar el modelo SPEROS-C en cuencas mediterráneas con una compleja topografía, litología y cobertura vegetal, con el fin explorar la potencialidad del modelo en este tipo de ambientes. El segundo objetivo (ii) consiste en evaluar y poner en perspectiva los valores de redistribución espacial de carbono orgánico dentro de la cuenca y su impacto en el balance de carbono. Para ello se tienen en cuenta tanto datos obtenidos con la aplicación del modelo como resultados de otros estudios.

\section{Zona de estudio}

El área de estudio pertenece a la cuenca de la Rogativa $\left(\sim 50 \mathrm{~km}^{2}\right.$, SE de España $\left.38^{\circ} 08^{\prime} \mathrm{N}, 2^{\circ} 13^{\prime} \mathrm{O}\right)$. Localizada al Oeste de la Región de Murcia, la Rogativa es una cuenca de tercer orden que drena al río Segura y posee un clima mediterráneo de transición entre el seco y subhúmedo con una temperatura media anual de $13.3^{\circ} \mathrm{C}$ y $581 \mathrm{~mm}$ de precipitación media anual. La cuenca posee una litología mixta de calizas y areniscas en las sierras que la limitan (Revolcadores y Cuerda de la Gitana) y relleno de margas en los fondos de valle (IGME, 1979). La mayoría de los suelos se clasifican como Regosoles Calcáricos, apareciendo también Leptosoles y Cambisoles Calcáricos (Alías et al., 1991). La cuenca estaba dominada por un paisaje eminentemente agrícola (cereales de secano) y forestal en los años 50, pero las restauraciones hidrológico-forestales que comenzaron a realizarse a partir del año 1975, junto con la coyuntura socioeconómica que condujo al éxodo rural, cambiaron definitivamente el patrón de usos del suelo en la cuenca. Así, en 1997 se detecta una reducción del área agrícola de más de del 50\% y un aumento del bosque por encima de ésta proporción (Boix-Fayos et al., 2007, 2008). Las restauraciones hidrológico-forestales realizadas consistieron en la construcción de una densa red de diques de retención de sedimentos en los cauces y la reforestación de sus áreas vertiente.

Este trabajo utiliza como base de datos para el estudio 12 de estas cuencas de drenaje delimitadas por diques de corrección hidrológica repartidas por toda la cuenca de la Rogativa (Fig. 1), de distintos tamaños y características (Tabla 1). Las cuencas de estudio ocupan distintas posiciones topográficas dentro de la cuenca de la Rogativa, recogen distintas litologías dominantes y muestran diferentes tamaños de área de drenaje con el fin de representar una gran parte de la variabilidad ambiental de la zona de estudio. De las 12 cuencas seleccionadas, 5 de ellas son unidades hidrológicas independientes y las 7 restantes cuentan con cuencas anidadas aguas arriba, aunque en estos casos sus datos han sido corregidos.

En la actualidad el paisaje de la Rogativa representa una mezcla de cereales de secano, plantaciones de nogales y viñedos de reciente implantación (Viña de la Rogativa desde aproximadamente 2007 perteneciente a la finca de Casas de Alfaro), bosques (principalmente Pinus nigra salzmanii, Pinus pinaster y Pinus halepensis) y matorrales (Erinacea anthyllis, Cytisus reverchonii, Rosmarinus officinalis, Thymus vulgaris y Genista scorpius). 
Figura 1. Localización de las 12 cuencas dentro de la zona de estudio (cuenca de la Rogativa).
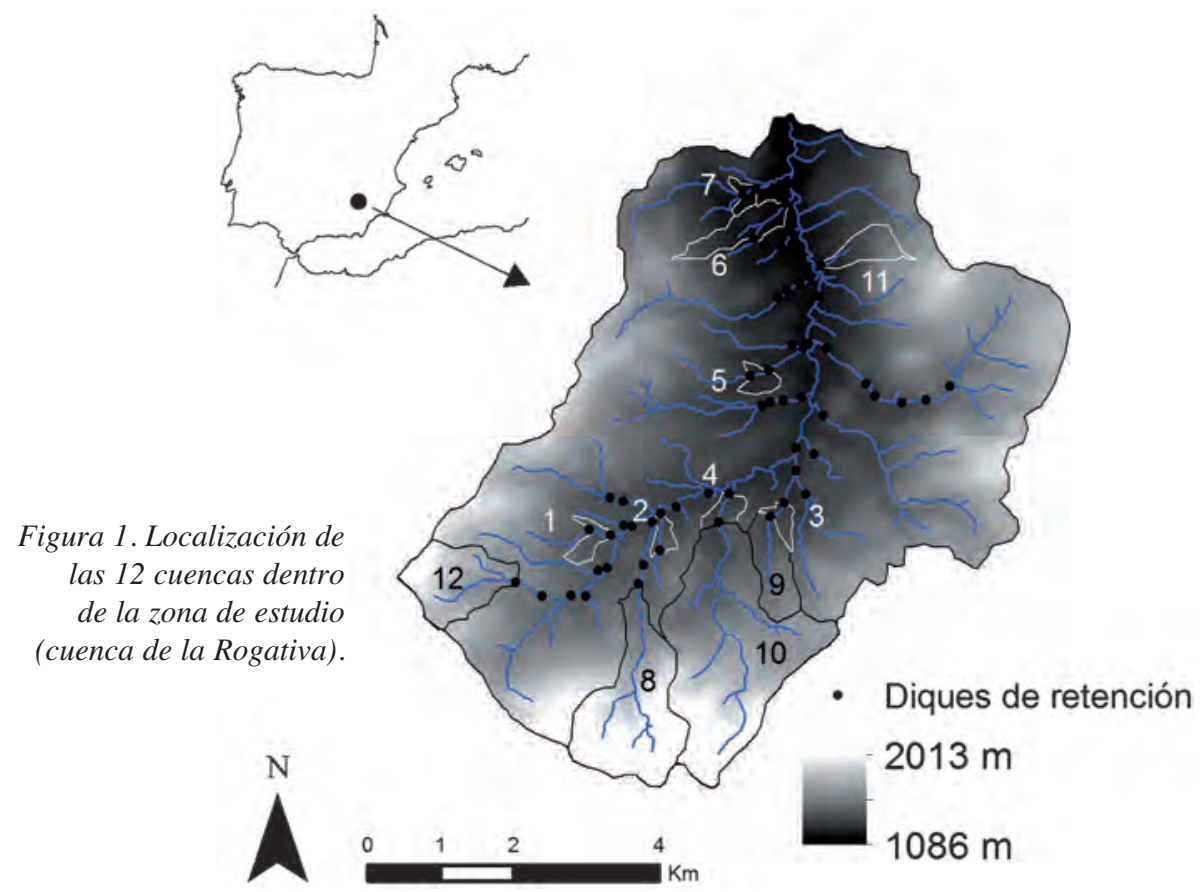

Tabla 1. Características principales de las 12 cuencas de estudio clasificadas según el tipo de litología dominante.

\begin{tabular}{|c|c|c|c|c|c|c|c|c|}
\hline $\begin{array}{l}\text { Litología } \\
\text { dominante }\end{array}$ & Id & $\begin{array}{l}\text { Área } \\
\text { (ha) }\end{array}$ & $\begin{array}{l}\text { Elevación } \\
\text { media }(m)\end{array}$ & $\begin{array}{l}\text { Pendiente } \\
\text { media }(\%)\end{array}$ & $\begin{array}{c}\% \mathrm{C} \text { en } \\
\operatorname{suelos}^{\mathrm{a}}(\%)\end{array}$ & $\begin{array}{c}\% \mathrm{C} \text { en } \\
\text { sedimentos }(\%)\end{array}$ & $\begin{array}{l}\mathrm{TE}^{\mathrm{b}} \\
(\%)\end{array}$ & $\begin{array}{c}\text { SYc } \\
\left(\text { Mg año }^{-1}\right)\end{array}$ \\
\hline \multirow[t]{8}{*}{ Margas } & 1 & 16.8 & 1320 & 16 & 1.3 & 1.2 & 88 & 9 \\
\hline & 2 & 12.3 & 1300 & 21 & 2.4 & 1.1 & 90 & 32 \\
\hline & 3 & 12.2 & 1276 & 26 & 1.6 & 1.0 & 92 & 16 \\
\hline & 4 & 16.1 & 1273 & 30 & 2.1 & 1.2 & 87 & 0 \\
\hline & 5 & 15.4 & 1221 & 20 & 2.2 & 1.0 & 94 & 75 \\
\hline & 6 & 44.4 & 1199 & 25 & 2.0 & 1.0 & 71 & 34 \\
\hline & 7 & 8.3 & 1153 & 24 & 2.5 & 1.1 & 96 & 3 \\
\hline & 8 & 37.8 & 1288 & 32 & 1.7 & 0.6 & 57 & 106 \\
\hline \multirow[t]{3}{*}{ Calizas } & 9 & 248.2 & 1709 & 46 & 2.0 & 1.6 & 15 & 79 \\
\hline & 10 & 68.6 & 1367 & 26 & 2.0 & 1.3 & 18 & 31 \\
\hline & 11 & 429.5 & 1547 & 34 & 2.2 & 1.4 & 28 & 207 \\
\hline $\begin{array}{l}\text { Cuaternario } \\
\text { indiferenciado }\end{array}$ & 12 & 120.1 & 1588 & 40 & 2.0 & 1.4 & 44 & 78 \\
\hline
\end{tabular}

${ }^{a}$ Concentración media de $\mathrm{C}$ en los suelos del área de drenaje calculado en base al patrón de usos del suelo del año 1981.

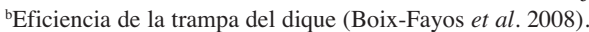

'Producción de sedimento (SY). 


\section{Métodos}

\subsection{Muestreo y análisis de suelo y sedimentos}

Los datos de suelo utilizados en este estudio proceden de un muestreo extenso de los suelos de toda la cuenca de la Rogativa realizado a lo largo de varios años. Para el mismo, se tomaron muestras repartidas entre los diferentes usos del suelo de la cuenca, muestreando los primeros $10 \mathrm{~cm}$ de suelo. En cada punto se tomaron muestras de volumen conocido $\left(100 \mathrm{~cm}^{3}\right)$ y muestra alterada para las determinaciones analíticas. En conjunto, se tomaron 120 muestras de suelo repartidas por toda la cuenca bajo los diferentes usos.

Las muestras de sedimento utilizadas en este estudio para las 12 cuencas se tomaron en un punto cercano a la pared del dique, en profundidad, tomando muestras de $100 \mathrm{~cm}^{3}$ a intervalos $(\sim 7 \mathrm{~cm})$ hasta que se alcanzaba la roca madre, aproximadamente de 1 a $1.5 \mathrm{~m}$ de profundidad. De esta manera se recogieron de promedio 15 muestras de sedimento por cuña de sedimentación (180 muestras de sedimento en total). Se disponía además de información de la producción de sedimentos a escala de subcuenca determinada en trabajos anteriores a partir del volumen de sedimentos retenidos y la densidad aparente de los mismos en las mismas cuencas (Boix-Fayos et al., 2008).

Las muestras de suelo alteradas se secaron al aire, se molieron y tamizaron a $2 \mathrm{~mm}$ para las determinaciones analíticas. Las muestras de suelo no alteradas se secaron a $105^{\circ} \mathrm{C}$ para la determinación de la densidad aparente. Las muestras de sedimentos se secaron a $60^{\circ} \mathrm{C}$ para la determinación de la densidad aparente, después se molieron y tamizaron a $2 \mathrm{~mm}$ para el resto de análisis. El contenido de carbono orgánico se determinó por el método de oxidación húmeda (Yeomans y Bremner, 1988) y por combustión seca en un analizador elemental (FLASH EA 1112 SERIES térmica). La distribución de tamaño de partícula se midió, tras dispersión química con calgón, a través de un método combinado que consistía en un tamizado en húmedo para la fracción de arena $(>63 \mu \mathrm{m})$ y medición por difractometría láser para las fracciones limo y arcilla $(<63 \mu \mathrm{m})$ utilizando un Coulter LS200 (Miami, EE.UU.), tras la eliminación de la materia orgánica por oxidación.

\subsection{El modelo SPEROS-C}

SPEROS-C simula el efecto de los procesos de erosión hídrica y/o por laboreo sobre el SOC y los flujos de C asociados a la movilización de sedimentos. Así, SPEROS-C modeliza tanto la redistribución (flujo lateral) de sedimentos y el C asociado a ellos a escala de paisaje, como los efectos de los flujos laterales de C sobre la mineralización y secuestro de $\mathrm{C}$ dentro de cada perfil (flujo vertical). El modelo SPEROS-C (Van Oost et al., 2005) consta de un componente de redistribución del suelo basado en el modelo de erosión SPEROS (Van Oost et al., 2003) y un componente dinámico de carbono orgánico del suelo basado en el modelo ICBM (Andrén y Kätterer, 1997). SPEROS-C, por lo tanto, integra el componente erosivo del suelo en la evolución del SOC a escala de ladera o de cuenca, todo ello a través de un enfoque de múltiples capas. La representación de las diferentes capas del suelo con sus tasas propias de reposición (tur- 
nover) de SOC permite la simulación de la dinámica de $\mathrm{C}$ en el perfil del suelo bajo el efecto de la deposición del SOC espacialmente redistribuido, y la simulación del efecto de la eliminación de la superficie del suelo en la dinámica de $\mathrm{C}$ en los perfiles de suelo erosionados (Van Oost et al., 2009). Aplicado a escala de cuenca, el modelo puede simular transferencias espaciales entre posiciones geomorfológicas y diferentes clases de cobertura y uso de suelo.

\subsubsection{Simulación de la redistribución del suelo}

La cuenca de la Rogativa es un buen ejemplo de un paisaje activo geomorfológicamente con cambios de uso documentados en su área de drenaje que han conducido a cambios en la dinámica fluvial, reflejados en la evolución de su morfología fluvial en los últimos 50 años (Boix-Fayos et al., 2007). Así pues cuenta con procesos erosivos activos y asumimos una redistribución de $\mathrm{C}$ en el paisaje asociada a los mismos. El patrón de usos del suelo queda organizado, en términos generales, con una distribución del bosque en las partes altas y medias de la cuenca, y una ubicación principal de las zonas agrícolas alrededor del cauce principal en las laderas adyacentes con pendientes más suaves.

Para el ejercicio de simulación con SPEROS-C se ha utilizado como escenario de uso de suelo, la cartografía de usos realizada a partir de la fotografía aérea digital de 1981 (IDERM, 2011) ya que era la más cercana disponible a la fecha en que se instalaron los diques de corrección hidrológica (1976-1977) y nos permite tener una idea aproximada del patrón de usos en el momento en que los diques comenzaron a recibir sedimentos. La cartografía de usos de suelo se ha realizado a partir de la digitalización manual del patrón de usos en cada una de las cuencas distinguiendo las siguientes cinco clases: bosque de elevada y baja densidad, matorral, pastizal y zona agrícola.

Como originalmente SPEROS-C fue creado para estudiar la redistribución de carbono en paisajes agrícolas, tuvimos que adaptar el modelo a las condiciones heterogéneas de nuestra zona de estudio. Esta adaptación comportó: a) desactivar el componente de laboreo, b) definir un sistema fluvial con capacidad de transporte infinita, c) definir una zona deposicional (cuña sedimentaria) con una eficiencia de trampa del 100\% (TE) a la salida de cada cuenca de drenaje, y, d) adaptar las ecuaciones de erosión del suelo y de capacidad de transporte de sedimentos (TC) a las de Verstraeten et al. (2007) para incluir el papel de una cobertura vegetal heterogénea y permitir mayor capacidad de transporte de sedimentos en áreas de acumulación de flujo. Por lo tanto, el componente de redistribución de suelo utilizado en SPEROS-C se basó en las Ecuaciones 1 y 2:

$$
\begin{aligned}
& E=L \cdot R \cdot K \cdot L S_{2 D} \cdot C \\
& T C=L \cdot K T C \cdot R \cdot K \cdot A^{1.4} \cdot S^{1.4}
\end{aligned}
$$

donde L es un parámetro de escala, $\mathrm{R}\left(\mathrm{MJ} \mathrm{mm} \mathrm{m}^{-2} \mathrm{~h}^{-1} \mathrm{año}^{-1}\right), \mathrm{K}\left(\mathrm{kg} \mathrm{MJ} \mathrm{h}^{-1} \mathrm{~mm}^{-1}\right)$ y C (-) representan los parámetros de la RUSLE de erosividad de la lluvia, erosionabilidad del suelo y el factor de cultivo, respectivamente. LS 2D representa el factor bidimensional topográfico (Desmet y Govers, 1996), A es el área drenaje $\left(\mathrm{m}^{2}\right)$, y S el gradiente de pendiente local $\left(\mathrm{m} \mathrm{m}^{-1}\right)$. KTC es el coeficiente de capacidad de transporte, que es específi- 
co según la cobertura vegetal y se utiliza para la calibración del modelo (Verstraeten et al., 2007). Se otorgó un valor bajo de KTc (KTc low) a superficies con densa cobertura vegetal y de un valor alto de KTc a áreas de poca vegetación y elevada capacidad de transporte de sedimentos.

Aunque, como se mencionó anteriormente, la eficiencia de trampa (TE) de la entrada a los diques se consideró del 100\% (con el fin de contabilizar todos los sedimentos y el C que alcanzan la principal zona de deposición), las salidas del modelo se corrigieron para una eficiencia de trampa estimada para cada dique (Boix-Fayos et al., 2008) con el fin de contabilizar de forma más realista las exportaciones de sedimento en el balance general de $\mathrm{C}$ en cada cuenca (Ecuación 3).

$$
C \text { exportado }=(C \text { en la cuña de sedimentos }) \cdot \frac{(100-T E)}{100}
$$

Finalmente, debido al desconocimiento de la dinámica del $\mathrm{C}$ depositado en las cuñas de sedimento, se consideró que no ocurrían procesos de mineralización sobre el C depositado allí y se utilizaron datos bibliográficos para cerrar el balance de $\mathrm{C}$ a escala de cuenca (ver más adelante).

\subsubsection{Simulación de la dinámica del carbono del suelo}

El componente de SOC se describe en el modelo de ICBM (Andrén y Kätterer, 1997) usando dos reservorios, uno joven (Y) y uno antiguo (O), las tasas de descomposición de los cuales dependen de un solo factor externo ' $r$ ' (adimensional ) que se define en la Ecuación 4:

$$
r=2.07^{\frac{T-5.4}{10}} \cdot \frac{1}{W l_{r}}
$$

donde $\mathrm{T}$ representa la temperatura media anual $\left(13.3^{\circ} \mathrm{C}\right)$ y $\mathrm{WI}$ el índice de humedad (Beven y Kirkby, 1979). Cuatro flujos principales controlan las reservas de SOC en cada grupo: (1) la entrada anual de $C$ en el suelo por biomasa aérea y subterránea, (2) la mineralización de $\mathrm{C}$ del reservorio joven, (3) el paso del $\mathrm{C}$ del reservorio joven al antiguo, $\mathrm{y}$ (4) y la mineralización de $\mathrm{C}$ del reservorio antiguo. La dinámica del carbono entre los dos reservorios se describe en las ecuaciones 5 y 6 :

$$
\begin{aligned}
& \frac{d Y}{d t}=i-k_{y} r Y \\
& \frac{d O}{d t}=h k_{y} r Y-K_{o} r O
\end{aligned}
$$

donde 'i' representa el promedio de entrada anual de carbono en el suelo $\left(\mathrm{kg} \mathrm{m}^{-2} \mathrm{año}^{-1}\right)$, ' $\mathrm{k}_{\mathrm{y}}$ ' $\mathrm{y}$ ' $\mathrm{k}_{\mathrm{o}}$ ' son las tasas de reposición (turnover) de $\mathrm{Y}$ y $\mathrm{O}$, respectivamente, que se establecieron a $0.8 \mathrm{y}^{-1}$ y $0.006 \mathrm{y}^{-1}$; ' $\mathrm{h}$ ' es el coeficiente de humificación (adimensional), es- 
tablecido a 0.125 y el coeficiente climático ' $r$ ' descrito anteriormente. Por simplificar, todos estos flujos verticales se calcularon asumiendo que los coeficientes de ' $h$ ' y los parámetros ' $\mathrm{k}_{\mathrm{y}}$ ' y ' $\mathrm{k}_{\mathrm{o}}$ ' no tienen variación espacial en nuestras cuencas y sus valores pueden ser directamente tomados de Andrén y Kätteren (1997).

\section{Resultados}

\subsection{Calibración y validación del modelo}

La combinación de parámetros de KTc high $4 * 10^{-4}$, KTc low $1.7 * 10^{-6}$ y parámetro de escala 1 resultó ser la calibración más adecuada, con un valor de eficiencia del modelo de 0.62 (Nash y Sutcliffe, 1970) para la estimación de la producción de sedimentos. El valor de $\mathrm{R}^{2}$ entre los valores observados y predichos fue de 0.70 . Con este modelo optimizado para la predicción de la producción de sedimentos, la eficiencia del modelo $(\mathrm{ME})$ y el coeficiente de determinación $\left(\mathrm{R}^{2}\right)$ para la exportación de carbono fueron 0.55 y 0.77 , respectivamente (Fig. 2).

Para la validación del modelo se aplicó el método Jackknife (Shao y Tu, 1995) que resultó en un valor de ME de 0.10 , un error cuadrático medio (RRMSE) de 0.95 y un $\mathrm{R}^{2}$ de 0.39 .

\subsection{Flujos de carbono inducidos por la erosión}

Para el escenario de uso de suelo de 1981, SPEROS-C predice tasas de movilización de C por erosión de suelo C entre 0.14 y $4.43 \mathrm{~g} \mathrm{~m}^{-2}$ año ${ }^{-1}$. Estos resultados se encuentran dentro del rango de las tasas medias anuales de movilización de $\mathrm{C}$ por erosión documentadas en otras cuencas pequeñas 0.05-23 gm-2 año $\mathrm{gm}^{-1}$ (Einsele et al., 2001;
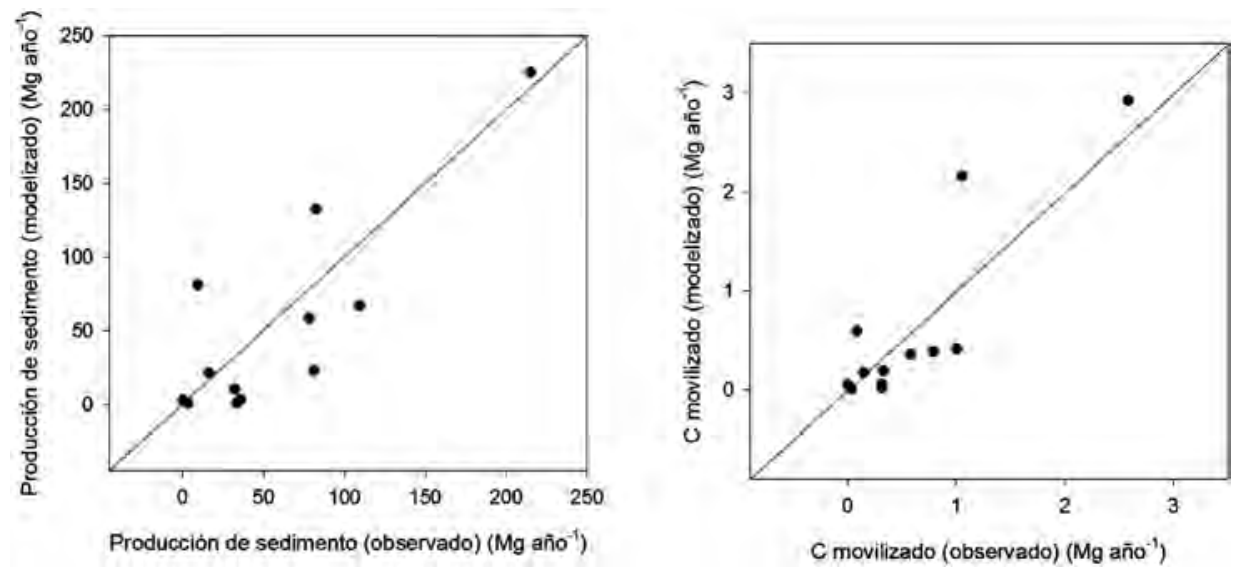

Figura 2. Gráficos de dispersión que muestran la producción de sedimento y movilización de $C$ observados versus los modelizados (se dibuja la línea 1:1 para referencia). 


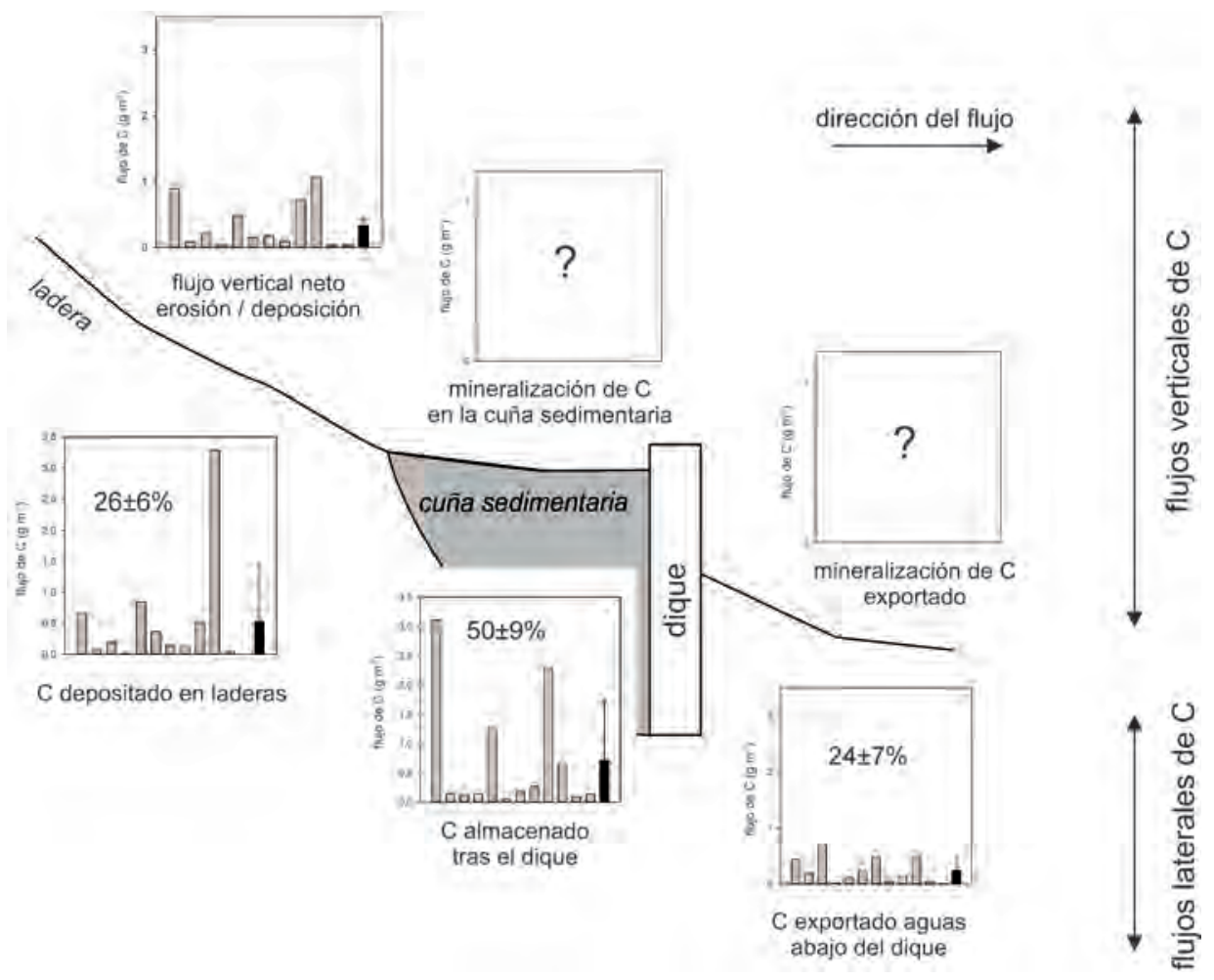

Figura 3. Flujos de $C$ inducidos por procesos erosivos. Se presenta el valor estimado para cada cuenca (en gris), y la media para toda la zona y su desviación (en negro). El porcentaje de C que llega a las cuñas sedimentarias también se incluye. Las interrogaciones indican que, a pesar de existir mineralización en las cuñas sedimentarias y durante la fase de transporte (tanto aguas arriba como aguas abajo del dique), estos flujos no se han considerado en la modelización. Los porcentajes en los gráficos de flujos laterales hacen referencia al destino de los flujos laterales de $C$.

Jacinthe et al., 2004; Moorman et al., 2004; Haregeweyn et al., 2008; Starr et al., 2008; Boix-Fayos et al., 2009; Juárez et al., 2011).

Una estimación de la exportación de sedimentos y $\mathrm{C}$ aguas abajo de los diques se obtuvo corrigiendo los sedimentos y $\mathrm{C}$ modelizados que llegan al dique por la eficiencia de la trampa del mismo (Ecuación 3). Estos datos indican que un promedio de $24 \pm 7 \%$ del C total movilizado por erosión y el $22 \pm 6 \%$ del total de suelo erosionado sobrepasa el dique y es exportado aguas abajo del mismo (Fig. 3). En la bibliografía se describe tanto más exportación de C (20\%) que de sedimento (10\%) (Smith et al., 2005), como más exportación de sedimentos que de C (4\% frente a $2 \%$ ) por erosión (Chaplot et al., 2005). El porcentaje de deposición de $\mathrm{C}$ en laderas modelizado $(26 \pm 6 \%)$ fue mayor que el valor de deposición $11-31 \%$ estimado por Yadav y Malanson (2009), pero inferior a la mayoría de los valores publicados previamente 
comprendidos entre 70-90\% (Stallard, 1998; Star, 2001; Chaplot et al., 2005; Smith et al., 2005; Van Oost et al., 2007).

La magnitud de los flujos verticales de carbono en una cuenca dependen del equilibrio entre las zonas de erosión y sedimentación (Dugloss et al., 2012). En nuestras cuencas, el flujo vertical medio de entrada de $\mathrm{C}$ en el suelos, tomando como referencia los valores del último año modelizado, fue de $0.35 \pm 0.11 \mathrm{~g} \mathrm{~m}^{-2}$, mientras que la emisión media de $\mathrm{C}$ a la atmósfera modelizada para el conjunto de la cuenca fue de $0.02 \pm 0.01 \mathrm{~g} \mathrm{~m}^{-2}$, provenientes de las zonas de deposición en las laderas (se da el valor medio y el error estándar en ambos casos). De este modo, los flujos netos asociados a los procesos de erosión resultan ser positivos en todos los casos, lo cual indica un secuestro neto de $\mathrm{C}$ a nivel de cuenca, aunque un orden de magnitud menor que los estimados en otros estudios utilizando el mismo modelo pero en ecosistemas agrícolas húmedos (Dugloss et al., 2012; Van Oost et al., 2005). Los flujos positivos verticales de carbono se incrementaron linealmente con el aumento de las tasas de erosión de $\mathrm{C}$ y se relacionan con el reemplazo dinámico de carbono en zonas erosionadas. En general, las zonas con una deposición neta de $\mathrm{C}$ se convirtieron en fuente de $\mathrm{CO}_{2}$ a la atmósfera por la eficiencia de los procesos de mineralización.

\section{Discusión}

\subsection{Aproximación al balance de carbono a escala de cuenca: integración de flujos laterales y verticales de carbono inducidos por procesos erosivos}

La integración de los flujos de $\mathrm{C}$ laterales y verticales proporciona una imagen general de los efectos de la erosión en el balance de $\mathrm{C}$ a la escala de las cuencas estudiadas. Todos los flujos de $\mathrm{C}$ considerados están representados y cuantificados para las 12 cuencas estudiadas (Fig. 3). En general, los flujos laterales de $\mathrm{C}$ fueron más importantes que los flujos de $\mathrm{C}$ verticales, ya que los últimos (balance neto entre negativos y positivos) representan menos de un tercio $(21 \pm 1 \%)$ del C movilizado por erosión. Este valor, similar al 23\% de reemplazo del C erosionado encontrado por Van Oost et al. (2007) con datos de zonas agrícolas de todo el mundo, indica que parte del C movilizado por erosión queda reemplazado de manera efectiva. Al mismo tiempo esto significa que la ocurrencia de secuestro neto de $\mathrm{C}$ depende del destino de $\mathrm{C}$ movilizado por erosión durante las fases de transporte y deposición.

Para el balance, se calcularon las tasas netas de secuestro de C para las 12 cuencas de dos formas: 1) combinando flujos verticales negativos y positivos, y 2) integrando flujos verticales y laterales. Con el primer enfoque se obtuvo un secuestro neto positivo medio de $\mathrm{C}$ para todas las cuencas de $0.33 \pm 0.10 \mathrm{~g} \mathrm{~m}^{-2}$, un valor más alto que el promedio exportado de $\mathrm{C}$ aguas abajo de los diques por los flujos laterales $\left(0.24 \pm 0.07 \mathrm{~g} \mathrm{~m}^{-2}\right)$. Sin embargo, estas estimaciones no incluyen los dos flujos adicionales necesarios para obtener una visión completa del proceso de mineralización de $\mathrm{C}$ durante el transporte y la mineralización de $\mathrm{C}$ tras la deposición (en la cuña de sedimentos propiciada por el dique en este caso). Las implicaciones de estos flujos se discuten a continuación. El 
segundo enfoque adoptado para el balance de $\mathrm{C}$ en el ciclo erosivo consideró los flujos del primer enfoque más la exportación de $\mathrm{C}$ aguas abajo del dique como componente negativo del balance, y el $\mathrm{C}$ almacenado en la cuña sedimentaria como un flujo positivo. Este segundo enfoque dio lugar a un secuestro neto, inducido por los procesos erosivos, de $0.80 \pm 0.04 \mathrm{~g} \mathrm{~m}^{-2}$. A pesar del secuestro positivo neto de $\mathrm{C}$ en general, tres de las cuencas se comportan como fuentes netas de $\mathrm{C}$ debido a la baja eficiencia de trampa de los diques situados a la salida de las mismas. En este sentido, la eficiencia como trampa de los diques desempeña un papel importante en estas estimaciones.

La mineralización de $\mathrm{C}$ durante el transporte y en la cuña de sedimentos no se modelizó en este estudio, suponiendo así que la mineralización de C durante la simulación afectó sólo al C erosionado depositado en laderas. En relación a la mineralización de $\mathrm{C}$ durante el transporte, algunos autores sugieren que ésta supone entre el 20-30\% de todo el C transportado (Jacinthe, 2001; Jacinthe et al., 2002; Lal, 2003). El aumento del flujo vertical de $\mathrm{C}$ durante el transporte, se atribuye a la rotura de agregados y está sujeto a un gran número de factores que pueden variar su importancia, tales como la humedad del suelo, la estructura y el grado de agregación de las partículas (Van Hemelryck et al., 2010). Sin embargo, la tasa de mineralización del 20-30\% ha sido cuestionada. En dos aplicaciones recientes de SPEROS-C en pequeñas cuencas agrícolas se concluyó que la mineralización durante el transporte tenía que ser inferior a este intervalo, ya que mediante el uso de los valores de 20-30\%, se observó una clara subestimación de las reservas (stocks) de C medidas en el campo en las zonas de deposición (Van Oost et al., 2005; Dugloss et al., 2012). Esto queda confirmado en nuestro estudio donde encontramos una buena concordancia entre el $\mathrm{C}$ medido y modelizado en las cuñas sedimentarias, sin tener en cuenta los flujos verticales durante el transporte. De esta manera, la incorporación de estos flujos verticales en la simulación llevaría a una subestimación significativa de $\mathrm{C}$ depositado tras los diques.

Todavía existe controversia sobre el efecto neto de la deposición de sedimentos y su entierro en la mineralización de C. Varios estudios concluyeron que el 20-50\% del C depositado es mineralizado (Jacinthe, 2001; Polyakov y Lal, 2004). Sin embargo, no se tiene información sobre el tiempo que necesita el C para ser mineralizado y cómo la tasa de mineralización se ve afectada por la subsiguiente deposición de sedimentos. Se ha sugerido que la tasa de mineralización en las zonas deposicionales disminuye debido a la formación de una costra superficial que protege el SOC del suelo subyacente y ralentiza la tasa de mineralización inicial en los sedimentos (Van Hemelryck et al., 2011). Los flujos de carbono por mineralización en zonas deposicionales también pueden disminuir cuando el material erosionado y depositado procede de capas más profundas del suelo con un contenido más bajo de C (Dugloss et al., 2012), o cuando una gran parte del C exportado es depositado y almacenado en condiciones anaeróbicas en los humedales (McCarty y Ritchie, 2002). Así en ambientes de rápida sedimentación como en las cuñas sedimentarias de las cuencas estudiadas, con sedimento y $\mathrm{C}$ asociados a diversas fuentes y procesos erosivos, y probablemente en estados y tasas de descomposición diferentes (Nadeu et al., 2012), es difícil predecir si habría un aumento del flujo inicial de C hacia 
la atmósfera tras la deposición, y qué tiempo transcurriría hasta que nuevo material depositado disminuyera esa tasa de mineralización.

La consideración de otros flujos verticales de C durante el transporte y la deposición sin duda podría cambiar el balance general de los flujos de $\mathrm{C}$ verticales en las cuencas estudiadas. Por ejemplo, si tomamos una tasa de mineralización del 5\% en transporte y una mineralización del 18\% tras la deposición (Van Hemelryck et al., 2010), el promedio de flujos verticales negativos asociados a estos dos procesos en las cuencas sería $0.20 \pm 0.07 \mathrm{~g} \mathrm{~m}^{-2}$, aumentando sustancialmente las tasas de mineralización asociadas a la deposición en laderas $\left(0.02 \pm 0.01 \mathrm{~g} \mathrm{~m}^{-2}\right)$, pero todavía inferiores en magnitud a las tasas medias positivas de las cuencas $\left(0.35 \pm 0.11 \mathrm{~g} \mathrm{~m}^{-2}\right)$. Sin embargo, el hecho de que el C modelizado acumulado detrás de los diques no sea significativamente diferente (MannWhitney test, $\mathrm{p}>0.1$ ) al medido en el campo (Fig. 2) sugiere que la consideración de estos flujos adicionales daría lugar a una subestimación del total de $\mathrm{C}$ almacenado. Desde una perspectiva diferente, la similitud entre las existencias de $\mathrm{C}$ modelizadas y medidas en las zonas deposicionales también podría indicar que la pérdida de $\mathrm{C}$ adicional por mineralización en las cuñas de sedimentos se compensa por la formación de $\mathrm{C}$ autóctono en las mismas, aunque la entrada de $\mathrm{C}$ autóctono se ha estimado de poca importancia desde la construcción de los diques hasta el momento (Nadeu et al., 2012).

\subsection{Evaluación global de SPEROS-C y necesidades de desarrollo futuro}

Actualmente existen sólo unos pocos modelos capaces de simular el efecto de la redistribución lateral de $\mathrm{C}$ sobre los flujos de verticales de $\mathrm{C}$ y las reservas de SOC a escala de cuenca. Debido a los muchos procesos que interactúan a la vez, los datos que requiere el modelo y las incertidumbres que ocasiona, muchos de los esfuerzos anteriores en este sentido han estado dirigidos a zonas con características ambientales bastante homogéneas con respecto a los suelos, vegetación y condiciones topográficas. Sin embargo, para hacer una evaluación realista del destino del carbono orgánico movilizado por erosión y bajo diferentes condiciones de uso del suelo, se precisa de la aplicación de este tipo de modelos en ecosistemas de mayor heterogeneidad espacial. Debido a estas incertidumbres, la interpretación de los resultados del modelo debe hacerse de manera cuidadosa y relativa. Por estas razones consideramos pertinente una evaluación crítica de los resultados de SPEROS-C, los supuestos de los que se partió y una valoración de alternativas de desarrollo futuro.

\subsubsection{Rendimiento del modelo}

Aplicaciones previas de SPEROS-C fueron calibradas y/o validadas mediante mediciones de ${ }^{137} \mathrm{Cs}$ (para sedimentos) o con mediciones de las reservas de SOC (para la dinámica del C) (Tabla 2). En este estudio calibramos el componente de redistribución de suelo SPEROS-C con valores de producción de sedimentos (SY), obteniendo una eficiencia (ME) de 0.60, un coeficiente de determinación $\left(\mathrm{R}^{2}\right)$ de 0.40 y un error medio cuadrático (RRMSE) de 0.63. El buen ajuste para $\mathrm{C}$ (ME de 0.55 y R ${ }^{2}$ de 0.77 ) con un modelo calibrado para sedimentos podría servir de validación del modelo. Además, se realizó otra validación utilizando el procedimiento Jackknife para SY que pro- 
porcionó resultados relativamente buenos para sedimentos, con valores de RRMSE y $\mathrm{R}^{2}$ dentro del rango de los obtenidos en otros estudios basados en las mismas o similares ecuaciones de erosión y de capacidad de transporte de sedimentos (Tabla 2).

Tabla 2. Valores de calibración (cal) y validación (val) para estudios que aplicaron los modelos SPEROS, SPEROS-C o Watem-Sedem (W-S) y, según corresponda, se proporciona la eficiencia del modelo (ME), el error cuadrático medio (RRMSE) y/o el coeficiente de determinación $\left(R^{2}\right)$.

\begin{tabular}{|c|c|c|c|c|c|c|}
\hline Referencia & Modelo & $\begin{array}{l}\text { Variable de } \\
\text { calibración }\end{array}$ & $\mathbf{N}$ & ME & RRMSE & $\mathbf{R}^{2}$ \\
\hline \multirow[t]{2}{*}{ Este estudio } & SPEROS-C cal & SY & 12 & 0.60 & 0.63 & 0.70 \\
\hline & val & SY & 12 & 0.10 & 0.95 & 0.39 \\
\hline Van Oost et al. 2003 & SPEROS cal & Cs & $\operatorname{pix}^{\mathrm{a}}$ & 0.55 & na & na \\
\hline Van Oost et al. 2005 & SPEROS-C val & Cs & pix $^{\mathrm{a}}$ & na & na & $0.40-0.45$ \\
\hline \multirow[t]{2}{*}{ Dlugoss et al. 2011} & SPEROS-C cal & $\mathrm{SOC}^{\mathrm{b}}$ & 65 & na & na & na \\
\hline & val & $\mathrm{SOC}^{\mathrm{c}}$ & pix $^{\mathrm{a}}$ & $-0.13-0.28$ & $0.31-0.70$ & $0.27-0.64$ \\
\hline \multirow[t]{2}{*}{ de Vente et al. 2008} & W-S & SSY $^{\mathrm{d}}$ & 61 & $0.09-0.62$ & na & 0.06 \\
\hline & val & $\mathrm{SSY}^{\mathrm{d}}$ & 61 & 0.35 & 0.96 & 0.48 \\
\hline Boix-Fayos et al. 2008 & W-S & SY & 17 & 0.84 & na & na \\
\hline Van Rompaey et al. 2005 & W-S & $\mathrm{SSY}^{\mathrm{d}}$ & 40 & 0.14 & na & 0.50 \\
\hline Verstraeten et al. 2007 & W-S & $\mathrm{SSY}^{\mathrm{d}}$ & 26 & 0.55 & 0.64 & na \\
\hline
\end{tabular}

${ }^{a}$ Todos los pixeles del raster fueron utilizados.

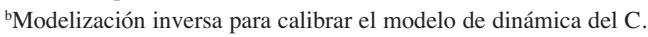

${ }^{\mathrm{c}}$ Comparación entre stocks de $\mathrm{C}$ modelizados y observados a distintas profundidades.

dProducción específica de sedimento (por unidad de área).

\subsubsection{Predicción de la erosión y redistribución de C}

SPEROS-C simula erosión hídrica y por laboreo en laderas, pero no en cárcavas y cauces. Al calibrar el modelo utilizando SY medida a escala de cuenca, esto podría causar una sobreestimación de las tasas de erosión y movilización de carbono en las laderas de las cuencas, donde la erosión concentrada y de los cauces forman una parte importante del balance de sedimentos (de Vente et al., 2008). Por lo tanto, se decidió añadir una red fluvial con infinita capacidad de transporte que permitió mayores tasas de transporte de sedimentos desde las laderas a la salida de las cuencas. Sin embargo, todavía queda por solucionar la ausencia de un componente de erosión del cauce que podría agregar importantes volúmenes de sedimentos de capas más profundas y por tanto más pobres en $\mathrm{C}$ que el material procedente de las laderas (Nadeu et al., 2012). Por otra parte, en ausencia de datos detallados sobre las prácticas de manejo agrícola de la zona, obviamos la movilización de carbono por la erosión debida al laboreo y nos centramos en el efecto de los procesos de erosión hídrica en todas las clases de uso de suelo. Somos conscientes de que la adición del componente de laboreo probablemente cambiaría los resultados de movilización de $\mathrm{C}$ por erosión en las zonas agrícolas y modificaría la 
importancia relativa de los flujos verticales asociados a la misma, como ocurre en otros estudios (Yadav y Malanson, 2009; Dugloss et al., 2012).

\subsubsection{Flujos verticales de $C$ durante el transporte y la sedimentación}

Los flujos verticales de carbono asociados a las fases de transporte y posterior deposición no se incluyeron en esta versión de SPEROS-C. Por lo tanto, nuestro balance de $\mathrm{C}$ se encuentra limitado por estos dos flujos de magnitud desconocida. Hemos planteado la hipótesis de su importancia relativa basada en resultados presentados en otros estudios y en nuestras propias evidencias. Sin embargo, la importancia de estos flujos a medio y largo plazo, especialmente los relacionados con el $\mathrm{C}$ enterrado en sumideros temporales tales como embalses o pantanos, sigue sin clarificar. Probablemente debido a la falta de consenso entre los resultados de campo y los de laboratorio, no tenemos constancia de que ninguno de los modelos disponibles en la actualidad pueda predecir el destino del $\mathrm{C}$ en zonas deposicionales o cuál es la influencia de las tasas de deposición de sedimentos en la dinámica del C en estos sitios. Las combinaciones de los modelos GeoWEPP o SWAT y CENTURY aplicadas por Yadav y Malanson (2009) y Yadav et al. (2009) y el modelo CREEP (Rosenbloom et al., 2001) no consideran la dinámica del $\mathrm{C}$ en las celdas de deposición de un modelo ráster, debido a una limitación del modelo CENTURY (versión 4.0). Una versión modificada del CENTURY 4.0 (Pennock y Frick, 2001) y el modelo EDEM (Liu et al., 2003) superan esta limitación del CENTURY, pero sólo simulan procesos en perfiles de suelo y por lo tanto no pueden ser utilizados a escala de paisaje; y SOrCERO (Billings et al., 2010) reproduce en detalle la dinámica de $\mathrm{C}$ pero sólo en los perfiles de erosión. SPEROS-C puede tener en cuenta los flujos de $\mathrm{C}$ verticales durante el transporte y en la fase de sedimentación, aunque los conceptos de los que parte pueden ser demasiado simples en áreas con elevadas tasas de acumulación de sedimentos, como las cuñas sedimentarias de la Rogativa, que se comportan de manera muy diferente a los suelos. Por lo tanto, un paso más en el desarrollo del modelo sería la consideración de la influencia del proceso de sedimentación en el C enterrado (McCarty et al., 2009), considerando la redistribución de los depósitos de $\mathrm{C}$ con diferentes tasas de reposición, en áreas con tasas elevadas de sedimentación.

\section{Conclusiones}

La modelización del carbono movilizado por erosión hídrica mostró que las 12 cuencas estudiadas fueron sumideros netos de $\mathrm{C}$ durante el periodo simulado (28 años). En contraste con otros estudios, la deposición de C en laderas representó sólo el $26 \%$ del total de $\mathrm{C}$ erosionado, mientras el resto se depositó en las cuñas sedimentarias tras los diques $(50 \%)$ o se exportó aguas abajo (24\%) de las mismas. Se carece de información directa sobre el destino del $\mathrm{C}$ atrapado por los diques y sobre los procesos de mineralización de $\mathrm{C}$ durante la fase de transporte, aunque el buen ajuste que presenta el modelo sugiere que estos procesos podrían ser de poca importancia en el área estudiada. En cuencas con este tipo de sumideros temporales (cuñas sedimentarias tras diques) el elevado porcentaje de carbono retenido en las mismas en comparación con el exportado 
fuera de ellas, y el depositado en laderas, sugiere que la estabilidad temporal del C depositado en estos sumideros es crucial para el balance de $\mathrm{C}$ en la cuenca.

\section{Agradecimientos}

Las muestras de suelo y sedimento utilizadas en la calibración y validación del modelo se obtuvieron y analizaron en el marco de los proyectos ERCO (CGL-200762590/BTE) y PROBASE (CGL2006-11619) del antiguo Ministerio de Ciencia e Innovación, y del proyecto ESUMA (11859/P/09) de la Fundación Séneca de la Región de Murcia y gracias a la colaboración de los compañeros y técnicos de laboratorio del Grupo de Erosión y Conservación de Suelos del CEBAS-CSIC. La aplicación del modelo fue posible gracias al apoyo logístico del Earth and Life Institute, en Louvain-laNeuve (Bélgica), y el apoyo económico de una estancia breve asociada a la beca FPI del antiguo Ministerio de Ciencia e Innovación (BES-2008-002379) concedida a E. Nadeu.

\section{Referencias bibliográficas}

Alías, L.J., Ortíz, R., Hernández, J., Martínez, J., Linares, D., Alcaraz, F., Sánchez, A., Marín, P. 1991. Proyecto LUCDEME. Mapa de suelos E=1:100 000 de la hoja de Caravaca-910. Ministerio de Agricultura, ICONA, Universidad de Murcia.

Andrén, O., Kätterer, T. 1997. ICBM: The introductory carbon balance model for exploration of soil carbon balances. Ecological Applications 7, 1226-1236.

Arnold, J.G., Srinivasan, R., Muttiah, R.S., Williams, J.R. 1998. Large area hydrologic modeling and assessment. Part I: Model development. Journal of the American Water Resources Association 34, 73-89.

Berhe, A.A., Harte, J., Harden, J.W., Torn, M.S. 2007. The significance of the erosion-induced terrestrial carbon sink. BioScience 57, 337-346.

Beven, K.J., Kirkby, M.J. 1979. A physically based variable contributing area model of basin hydrology / Un modèle à base physique de zone d'appel variable de l'hydrologie du bassin versant. Hydrological Sciences Bulletin 24, 43-69.

Billings, S.A., Buddemeier, R.W., Richter, D.D., Van Oost, K., Bohling, G. 2010. A simple method for estimating the influence of eroding soil profiles on atmospheric $\mathrm{CO}_{2}$. Global Biogeochemical Cycles 24, GB2001.

Boix-Fayos, C., Barberá, G.G., López-Bermúdez, F., Castillo, V.M. 2007. Effects of check dams, reforestation and land-use changes on river channel morphology: Case study of the Rogativa catchment (Murcia, Spain). Geomorphology 91, 103-123.

Boix-Fayos, C., de Vente, J., Martínez-Mena, M., Barberá, G.G., Castillo, V. 2008. The impact of land use change and check-dams on catchment sediment yield. Hydrological Processes 22, 4922-4935.

Boix-Fayos, C., de Vente, J., Albaladejo, J., Martínez-Mena, M. 2009. Soil carbon erosion and stock as affected by land use changes at the catchment scale in Mediterranean ecosystems. Agriculture, Ecosystems \& Environment 133, 75-85.

Chaplot, V.A.M., Rumpel, C., Valentin, C. 2005. Water erosion impact on soil and carbon redistributions within uplands of Mekong River. Global Biogeochemical Cycles 19, GB4004.

De Vente, J., Poesen, J., Verstraeten, G., Van Rompaey, A., Govers, G. 2008. Spatially distributed modelling of soil erosion and sediment yield at regional scales in Spain. Global and Planetary Change 60, 393-415. 
Desmet, P.J.J., Govers, G. 1996. A GIS procedure for automatically calculating the USLE LS factor on topographically complex landscape units. Journal of Soil and Water Conservation $51,427-433$.

Dlugoss, V., Fiener, P., Van Oost, K., Schneider, K. 2012. Model based analysis of lateral and vertical soil carbon fluxes induced by soil redistribution processes in a small agricultural catchment. Earth Surface Processes and Landforms37, 193-208.

Einsele, G., Yan, J., Hinderer, M. 2001 . Atmospheric carbon burial in modern lake basins and its significance for the global carbon budget. Global and Planetary Change 30, 167-195.

Flanagan, D.C., Nearing, M.A. 1995. USDA - Water Erosion Prediction Project (WEPP) Hillslope Profile and Watershed Model Documentation. USDA- Agricultural Research Service, NSERL Report No. 10. National Soil Erosion Research Laboratory, West Lafayette, Indiana.

Gregorich, E.G., Greer, K.J., Anderson, D.W., Liang, B.C. 1998. Carbon distribution and losses: Erosion and deposition effects. Soil \& Tillage Research 47, 291-302.

Harden, J.W., Sharpe, J.M., Parton, W.J., Ojima, D.S., Fries, T.L., Huntington, T.G., Dabney, S.M. 1999. Dynamic replacement and loss of soil carbon on eroding cropland. Global Biogeochemical Cycles 13, 885-901.

Haregeweyn, N., Poesen, J., Deckers, J., Nyssen, J., Haile, M., Govers, G., Verstraeten, G., Moeyersons, J. 2008. Sediment-bound nutrient export from micro-dam catchments in Northern Ethiopia. Land Degradation \& Development 19, 136-152.

Infraestructura de datos espaciales de la Región de Murcia (IDERM) 2011. Ortofoto regional del vuelo de 1981. Diponible en: http://cartomur.imida.es/visorcartoteca/ (fecha de acceso: 20/06/2011).

Instituto Geológico y Minero de España (IGME) 1979. Mapa geológico de España 1:50000. Nerpio 909, 23-26.

Jacinthe, P.A. 2001. Assessing Water Erosion Impacts on Soil Carbon Pools and Fluxes. En Assessment Methods for Soil Carbon. Advances in Soil Science, R. Lal (ed.), CRC Press, Boca Raton, Florida, pp. 427-449.

Jacinthe, P.A., Lal, R., Kimble, J.M. 2002. Carbon dioxide evolution in runoff from simulated rainfall on long-term no-till and plowed soils in southwestern Ohio. Soil \& Tillage Research 66, 23-33.

Jacinthe, P.A., Lal, R., Owens, L.B., Hothen, D.L. 2004. Transport of labile carbon in runoff as affected by land use and rainfall characteristics. Soil \& Tillage Research 77, 111-123.

Juárez, S., Rumpel, C., Mchunu, C., Chaplot, V. 2011. Carbon mineralization and lignin content of eroded sediments from a grazed watershed of South-Africa. Geoderma 167-168, 247-253.

Lal, R. 2003. Soil erosion and the global carbon budget. Environment International 29, 437-450.

Liu, S.G., Bliss, N., Sundquist, E., Huntington, T.G. 2003. Modeling carbon dynamics in vegetation and soil under the impact of soil erosion and deposition. Global Biogeochemical Cycles 17, 1074.

McCarty, G.W., Ritchie, J.C. 2002. Impact of soil movement on carbon sequestration in agricultural ecosystems. Environmental Pollution 116, 423-430.

McCarty, G., Pachevsky, Y., Ritchie, J. 2009. Impact of sedimentation on wetland carbon sequestration in an agricultural watershed. Journal of Environmental Quality 38, 804-813.

Moorman, T.B., Cambardella, C.A., James, D.E., Karlen, D.L., Kramer, L.A. 2004. Quantification of tillage and landscape effects on soil carbon in small Iowa watersheds. Soil \& Tillage Research 78, 225-236.

Nadeu, E., Berhe, A.A., de Vente, J., Boix-Fayos, C. 2012. Erosion, deposition and replacement of soil organic carbon in Mediterranean catchments: a geomorphological, isotopic and land use change approach. Biogeosciences 9, 1099-1111.

Nash, J.E., Sutcliffe, J.V. 1970. River flow forecasting through conceptual models part I - A discussion of principles. Journal of Hydrology 10, 282-290. 
Parton, W.J., Schimel, D.S., Cole, C.V., Ojima, D.S. 1987. Analysis of factors controlling soil organic matter levels in Great Plains grasslands. Soil Science Society of America Journal 51, 1173-1179.

Pennock, D.J., Frick, A.H. 2001. The role of field studies in landscape-scale applications of process models: an example of soil redistribution and soil organic carbon modeling using CENTURY. Soil \& Tillage Research 58, 183-191.

Polyakov, V.O., Lal, R. 2004. Soil erosion and carbon dynamics under simulated rainfall. Soil Science 169, 590-599.

Renschler, C.C. 2003. Designing geo-spatial interfaces to scale process modes: the GeoWEPP approach. Hydrological Processes 17, 1005-1017.

Rosenbloom, N.A., Doney, S.C., Schimel, D.S. 2001. Geomorphic evolution of soil texture and organic matter in eroding landscapes. Global Biogeochemical Cycles 15, 365-381.

Schlesinger, W.H. 1990. Evidence from chronosequence studies for a low carbon-storage potential of soils. Nature 348, 232-234.

Shao, J.X., Tu, D. 1995. The Jackknife and Bootstrap. Springer, New York.

Smith, S.V., Sleezer, R.O., Renwick, W.H., Buddemeier, R. 2005. Fates of eroded soil organic carbon: Mississippi basin case study. Ecological Applications 15, 1929-1940.

Stallard, R.F. 1998. Terrestrial sedimentation and the carbon cycle: Coupling weathering and erosion to carbon burial. Global Biogeochemical Cycles 12, 231-257.

Starr, B. 2001. Assessing the Impact of Erosion on Soil Organic Carbon Pools and Fluxes. En Assessment Methods for Soil Carbon. Advances in Soil Science, R. Lal (ed), CRC Press, Boca Ratón, Florida, pp. 403-416.

Starr, G.C., L, R., Owens, L., Kimble, J. 2008. Empirical relationships for soil organic carbon transport from agricultural watersheds in Ohio. Land Degradation \& Development 19, 57-64.

Van Hemelryck, H., Fiener, P., Van Oost, K., Govers, G., Merckx, R. 2010. The effect of soil redistribution on soil organic carbon: An experimental study. Biogeosciences 7, 3971-3986.

Van Hemeltyck, H., Govers, G., Van Oost, K., Merckx, R. 2011. Evaluating the impact of soil redistribution on the in situ mineralization of soil organic carbon. Earth Surface Process and Landforms 36, 427-438.

Van Oost, K., Govers, G., Van Muysen, W. 2003. A process-based conversion model for caesium137 derived erosion rates on agricultural land: An integrated spatial approach. Earth Surface Processes and Landforms 28, 187-207.

Van Oost, K., Govers, G., Quine, T.A., Heckrath, G., Olesen, J.E., De Gryze, S., Merckx, R. 2005. Landscape-scale modeling of carbon cycling under the impact of soil redistribution: The role of tillage erosion. Global Biogeochemical Cycles 19, 1733-1739.

Van Oost, K., Quine, T.A., Govers, G., De Gryze, S., Six, J., Harden, J.W., Ritchie, J.C., McCarty, G.W., Heckrath, G., Kosmas, C., Giraldez, J.V., Da Silva, J.R.M., Merckx, R. 2007. The impact of agricultural soil erosion on the global carbon cycle. Science 318, 626-629.

Van Oost, K., Cerdan, O., Quine, T.A. 2009. Accelerated sediment fluxes by water and tillage erosion on European agricultural land. Earth Surface Processes and Landforms 34, 1625-1634.

Verstraeten, G., Prosser, I.P., Fogarty, P. 2007. Predicting the spatial patterns of hillslope sediment delivery to river channels in the Murrumbidgee catchment, Australia. Journal of Hydrology $334,440-454$.

Yadav, V., Malanson, G.P. 2009. Modeling impacts of erosion and deposition on soil organic carbon in the Big Creek Basin of southern Illinois. Geomorphology 106, 304-314.

Yadav, V., Malanson, G.P., Bekele, E., Lant, C. 2009. Modeling watershed-scale sequestration of soil organic carbon for carbon credit programs. Applied Geography 29, 488-500.

Yeomans, J.C., Bemmer, J.M. 1988. A rapid and precise method for routine determination of organic carbon in soil. Communications in Soil Science \& Plant Analysis 19, 1467-1476. 